Dengqiu Ma

Zhenhuan Ye

Hang Yang

https://doi.org/10.21278/TOF.4310]

ISSN 1333-1124

eISSN 1849-1391

\title{
TOOTH SURFACE RECONSTRUCTION AND TOOTH PROFILE GEOMETRIC ANALYSIS OF CIRCULAR ARC TOOTH TRACE CYLINDRICAL GEARS
}

\begin{abstract}
Summary
To reconstruct the tooth surface of a circular arc tooth trace cylindrical gear (CATT gear), one needs to create an accurate 3D model and investigate geometric features of the tooth profile. According to the processing principle of the rotating knife dish milling, and using the space meshing theory and differential geometry, the tooth surface equation and the tooth profile of CATT gears have been deduced. Then, the tooth surface reconstruction of CATT gears was done and a 3D model was created. Next, based on theoretical deduction, it was proven that the profile of the middle section was involute, and the other section was hyperbolic. Finally, the influences of the design parameters on the tooth profile were investigated. The general tendency is that the gear tooth thickness becomes thinner as the gear width increases and the tooth line radius decreases, and the tooth profiles of the concave and the convex tooth surface may intersect. The influences of the module need to be discussed separately. The tooth thickness deviation increases as the module increases. But the relative tooth thickness deviation is opposite. Therefore, it is suggested that $R_{T} \geq(3-4) b$ and the module should be large to improve the bearing capacity. The results of the investigation provide a reference for the design, profile modification, lubrication, and measuring of CATT gears.
\end{abstract}

Key words: $\quad$ circular arc tooth trace cylindrical gear; tooth surface reconstruction; tooth profile; tooth line radius; gear width; module

\section{Introduction}

With the development of equipment, the requirement for good meshing performance becomes increasingly strict for gear transmission [1]. To achieve good performance, scholars attempt to modify the tooth profile and the tooth line [2]. Therefore, it has been proposed to use a new type of gear transmission. i.e. the circular arc tooth trace cylindrical gears (CATT gears). It has characteristics of traditional gear transmission, and its line of action is a space curve, which avoids the initial instantaneous mesh impact [3]. The tooth line along the teeth width is a spatial arc, on the one hand, making the contact line longer than in the spur gear and resulting in CATT gears having better carrying capacity; on the other hand, it makes the tooth shape become an arc and the tooth width becomes wider, which results in CATT gears 
having higher bending strength [4]. CATT gears can overcome the disadvantages of spur gears, helical gears and herringbone gears. Therefore, CATT gears can be used in the field of aviation and aerospace in which gears need to meet the requirements of high speed transmission, carry heavy loads, be stable, produce low noise and have high reliability [5]. Many studies indicate that the application of CATT gears is very promising [6-7].

Numerous valuable studies have been carried out by scholars to investigate CATT gears. As for the manufacturing methods, the mainstream methods to cut CATT gears are the rotating knife dish milling processing and the parallel translating mechanism cutting processing [8-10]. But the characteristics of the tooth surface are different depending on the manufacturing method used. The gear cut by using a parallel translating mechanism is called ideal CATT gear. In the ideal CATT gear, tooth space width and tooth thickness are equal, and the gear's whole tooth profile is involute. However, its stiffness, efficiency and machining accuracy are unsatisfactory, so it cannot be applied in actual production [7]. CATT gears can also be cut by using rotating knife dish milling [9-11]. In this case, its tooth thickness and tooth space are equal in the middle cross section, but not equal in other sections, because the gear's tooth profiles are different in different sections. Papers [10-11] reveal that the CATT gear's tooth profile is involute in the middle section, other sections are hyperbolae, but mathematical proof is not provided in the papers. Comparing the two processing methods, the rotating knife dish milling processing is superior, for its structure has enough stiffness, and the processing method has high efficiency, machining accuracy, and the machine equipment can be modified by using the existing machine tools. However, CATT gears cut by using the rotating knife dish milling still need to be further studied for the purposes of application, design, etc. This paper presents current investigation into CATT gears processed by applying the rotating knife dish milling.

Rui-Tang Tseng [12] investigated the undercutting conditions, transmission errors and bearing contact of CATT gears under different assembly conditions by using Latvian's method [13-14]. Chen Ming and Di Yutao [15] investigated the forming process meshing interference, axis error and meshing influence caused by the centre distance, but they did not discuss the effect of related parameters on the objects of study. Ma Zhenqun [16] proposed a generating method with a tilted double-sided cutter to modify the whole tooth surface of cylindrical gears with arcuate tooth trace for the modification control question. However, he did not investigate the influence of the design parameters. He also did not propose a method to determine the amount of modification of the whole tooth surface for different parameters. Therefore, the method for modifying the whole tooth surface still needs to be further studied for the purposes of application, design, etc. Xiao Huajun [10] established a mathematical model to create a 3D model using Maple. The gear had high surface quality, but the author neither introduced a selection of parameters and a forming process nor analyzed the tooth surface geometric features. Jiang Weixu [17] and Wang Shaojiang [9] realized the CATT gear's simulation processing by using a rack cutter in UG NX8.0, but the surface of the CATT gear is rough, and the model could not be used for analysis and processing. Many researchers investigated the contact strength, bending strength, gear transmission errors, computerized design and simulation of meshing by applying the finite element analysis [18-21].

To apply CATT gears as soon as possible, the authors will deduce the tooth surface equation and the tooth profile equation based on the theories of differential geometry and meshing. Then, on this basis, the tooth surface of the CATT gear will be reconstructed. In addition, the authors will discuss the tooth profile features and analyze the influence of the modulus $m$, tooth line radius $R_{T}$ and gear width $b$ on the tooth thickness. This paper will contribute to the gear design and manufacture. 


\section{Mathematical model}

\subsection{Forming of CATT gears with a the rotary milling cutter}

The rotating milling processing device is shown in Fig. 1. The rotary knife dish is installed on the machine spindle and revolves around it. The double cutting edge tools are installed and fixed onto the edge of the circular knife dishes, and they rotate with it. The distance between the cutter axis and the machine spindle axis is $R_{T} . \alpha$ (pressure angle) is an angle between the internal and the external cutting edge, the cutter axis and the knife dish axis. When the knife dish rotates periodically, two cones of the same size and the opposite cone formed from the internal and the external cutting edge, two cones are used to form the concave and the convex tooth surface. As for the movement of the workpiece, the workpiece rotates around its own axis and it also moves forth and back in the horizontal direction. The tooth surface can be formed cooperatively by the workpiece movement and the knife dish movement. When the tooth is cut completely, the workpiece rotates to the next position for cutting until the whole gear is cut. This processing method can cut the concave and the convex tooth surface at the same time, and it can ensure the accuracy of machining the tooth shape as well as processing efficiency.

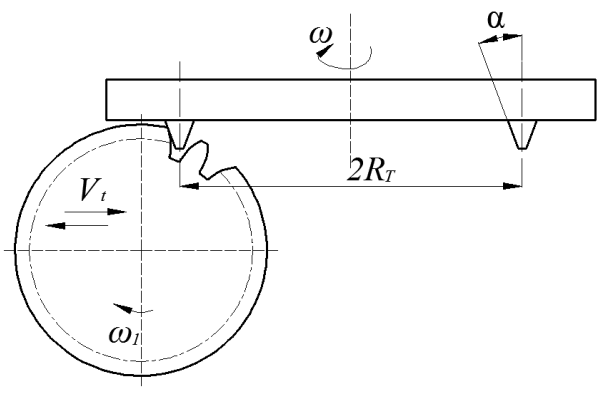

Fig. 1 Rotating milling processing device

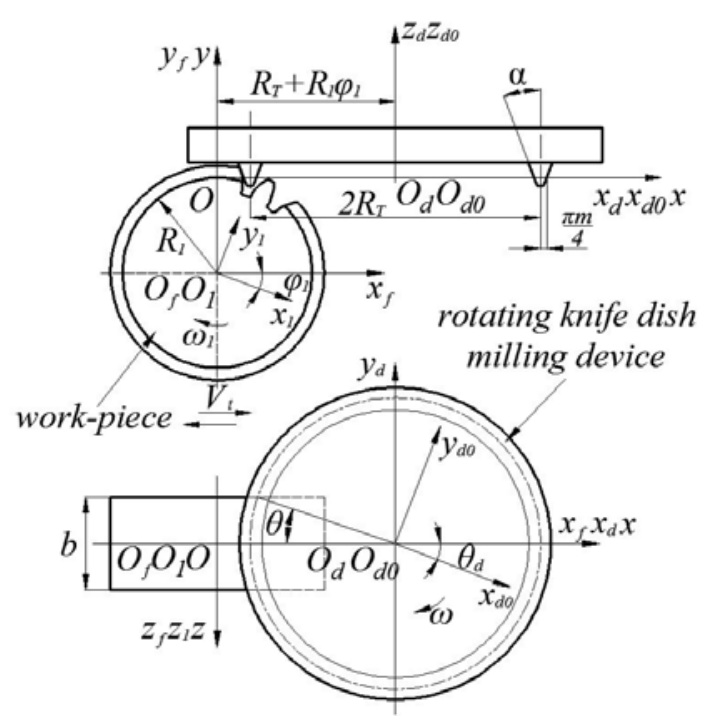

Fig. 2 Reference coordinate system

\subsection{Coordinate systems}

To study the forming process and the geometrical relationship of a CATT gear processed by using a rotating knife dish milling device, a coordinate system is set up in the processing system as shown in Fig. 2. $S_{d 0}\left(O_{d 0} X_{d 0} Y_{d 0} Z_{d 0}\right)$ (written as $\left.S_{d 0}\right)$ is built and fixed onto the rotating knife dish milling device, $O_{d 0}$ is located at the centre of the rotating knife dish, $O_{d 0} Z_{d 0}$ is located along the axis of the rotating knife dish, $S_{d 0}$ rotates with the rotation of the rotating knife dish. In other words, the cutting tool is stationary relative to $S_{d 0}$. $S_{d}\left(O_{d} X_{d} Y_{d} Z_{d}\right)$ (written as $\left.S_{d}\right)$ is also built on the rotating knife dish milling device, $O_{d}$ and $O_{d} Z_{d}$ also have the same position as $O_{d 0}$ and $O_{d 0} Z_{d 0}$, but $S_{d}$ is fixed and stationary relative to the world coordinate system. $S_{1}\left(O_{1} X_{1} Y_{1} Z_{1}\right)$ (written as $\left.S_{1}\right)$ is built and fixed onto the workpiece, $O_{1}$ is located at the centre of the workpiece, $O_{1} Z_{1}$ is located along the axis of the workpiece, the workpiece is stationary relative to $S_{1}$ the same as $S_{d 0} . S(O X Y Z)$ (written as $S$ ) is built on the workpiece, it is fixed and stationary relative to the world coordinate system, $O Y$ is parallel to 
$O_{d} Z_{d}, O Z$ has the same position as $O_{1} Z_{1}$, but $O$ is located at the intersection point between the pitch circle and $O Z . S_{f}\left(O_{f} X_{f} Y_{f} Z_{f}\right)$ (written as $S_{f}$ ) is an assistant coordinate system. The directions of $O_{f} X_{f}, O_{f} Y_{f}, O_{f} Z_{f}$ are the same as with $S$, just $O_{f}$ is located at the centre of the workpiece. In Fig. 2, $R_{1}$ is the pitch circle of the gear blank; $\varphi_{1}$ is the rotation angle of the gear blank; $R_{T}$ is the cutter radius (tooth line radius); $\theta$ is the rotation angle measured from the cutter to the end face of the gear blank in the process of machining and called tooth profile position angle, and the clockwise direction is positive.

The tooth surface of the CATT gears is enveloped by the cutter edge. Therefore, in the derivation process of the tooth surface, the coordinate transformation is needed from $S_{d 0}$ to $S_{1}$ base on the space geometry. The transformation matrix $M_{1}$ from $S_{d 0}$ to $S_{d}$ is represented as

$$
M_{1}=\left[\begin{array}{cccc}
\cos \theta & \sin \theta & 0 & 0 \\
-\sin \theta & \cos \theta & 0 & 0 \\
0 & 0 & 1 & 0 \\
0 & 0 & 0 & 1
\end{array}\right]
$$

The transformation matrix $M_{2}$ from $S_{d}$ to $S$ can be represented as

$$
M_{2}=\left[\begin{array}{cccc}
1 & 0 & 0 & R_{1} \varphi_{1}+R_{T} \\
0 & 0 & 1 & 0 \\
0 & -1 & 0 & 0 \\
0 & 0 & 0 & 1
\end{array}\right]
$$

The transformation matrix $M_{3}$ from $S$ to $S_{f}$ can be represented as

$$
M_{3}=\left[\begin{array}{cccc}
1 & 0 & 0 & 0 \\
0 & 1 & 0 & R_{1} \\
0 & 0 & 1 & 0 \\
0 & 0 & 0 & 1
\end{array}\right]
$$

The transformation matrix $M_{4}$ from $S_{f}$ to $S_{1}$ can be represented as

$$
M_{4}=\left[\begin{array}{cccc}
\cos \varphi_{1} & -\sin \varphi_{1} & 0 & 0 \\
\sin \varphi_{1} & \cos \varphi_{1} & 0 & 0 \\
0 & 0 & 1 & 0 \\
0 & 0 & 0 & 1
\end{array}\right]
$$

Therefore, the transformation matrix $M$ from $S_{d 0}$ to $S_{1}$ can be represented as

$$
M=M_{4} M_{3} M_{2} M_{1}
$$

2.3 Shape of the cross section and the generatrix equation of the cutting tool

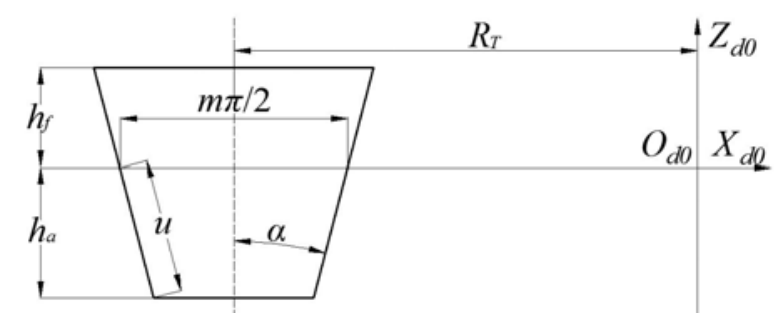

Fig. 3 Geometry and parameters of the cutter edge with sharp corners 
In gear machining, the tool geometry has a big influence on the forming of the gear tooth surface. In normal conditions, the tooth surface equation depends on the tool geometry and the relative movement relationship between the cutter and the workpiece. Usually, the effective cross section of the cutting tool is used to study the gear forming process. There are two situations when the geometry parameter of the cutting tool is measured [8]. Some studies regard the cross section of the cutting tool as tooth profile with sharp corners; some other studies regard the cross section of the cutting tool as tooth profile with rounded corners. For simplification purposes, the cutting tool with sharp corners is here adopted to study the tooth surface equation, shown in Fig. 3. $h_{f}$ is the gear root height of the cutter, $h_{a}$ is the gear top height of the cutter, $u$ is the displacement of a point from the blade to the reference point.

According to Fig. 3, the radii of the internal and external cutter's edges on the $X$ axis can be represented as

$$
R_{i 1}=R_{T}-\frac{m \pi}{4}, R_{o 1}=R_{T}+\frac{m \pi}{4}
$$

where $R_{i 1}$ and $R_{o 1}$ are the radii of the internal cutter's edge inside and the external cutter's edge, respectively.

And, any point on the cross section of the internal and external cutter's edge can be represented as in $S_{d 0}$

$$
\left\{\begin{array}{l}
x_{d 0}=-\left( \pm u \sin \alpha+R_{T} \pm \frac{m \pi}{4}\right) \\
y_{d 0}=0 \\
z_{d 0}=u \cos \alpha
\end{array}\right.
$$

According to the coordinate transformation, any point on the cross section of the internal and external cutter's edge can be represented as in $S_{d}$

$$
\left\{\begin{array}{l}
x_{d}=-\left( \pm u \sin \alpha+R_{T} \pm \frac{m \pi}{4}\right) \cos \theta \\
y_{d}=\left( \pm u \sin \alpha+R_{T} \pm \frac{m \pi}{4}\right) \sin \theta \\
z_{d}=u \cos \alpha
\end{array}\right.
$$

Eq. (8) can be written as a vector forming

$$
\boldsymbol{r}_{d}=-\left( \pm u \sin \alpha+R_{T} \pm \frac{m \pi}{4}\right) \cos \theta \boldsymbol{i}_{d}+\left( \pm u \sin \alpha+R_{T} \pm \frac{m \pi}{4}\right) \sin \theta \boldsymbol{j}_{d}+u \cos \alpha \boldsymbol{k}_{d}
$$

where $\boldsymbol{i}, \boldsymbol{j}, \boldsymbol{k}$ are the unit vectors along the $X, Y$ and $Z$ axes; the vector subscript represents the coordinate system (throughout the paper as well).

In order to study the contact line between the cutter and the workpiece, the tangent vectors of the cutter's revolving surface in the direction of $u$ and $\theta$ are represented based on the geometric principle and $\boldsymbol{r}_{d}$

$$
\begin{aligned}
& \frac{\delta \boldsymbol{r}_{d}}{\delta \theta}=\left( \pm u \sin \alpha+R_{T} \pm \frac{m \pi}{4}\right) \sin \theta \boldsymbol{i}_{d}+\left( \pm u \sin \alpha+R_{T} \pm \frac{m \pi}{4}\right) \cos \theta \boldsymbol{j}_{d} \\
& \frac{\delta \boldsymbol{r}_{d}}{\delta u}= \pm u \sin \alpha \cos \theta \boldsymbol{i}_{d} \pm u \sin \alpha \sin \theta \boldsymbol{j}_{d}+\cos \theta \boldsymbol{k}_{d}
\end{aligned}
$$


And, the normal vector of the cutter's revolving surface is further represented as

$$
\boldsymbol{n}_{d}=\frac{\delta \boldsymbol{r}_{d}}{\delta \theta} \times \frac{\delta \boldsymbol{r}_{d}}{\delta u}=\left( \pm u \sin \alpha+R_{T} \pm \frac{m \pi}{4}\right)\left(-\cos \alpha \cos \theta \boldsymbol{i}_{d}+\sin \alpha \sin \theta \boldsymbol{j}_{d} \mp \sin \alpha \boldsymbol{k}_{d}\right)
$$

\subsection{Tooth surface equation}

The machining processing of CATT gears could be regarded as the meshing movement of the basic rack and the workpiece, and the tooth surface is enveloped by the cutter tooth surface [22]. According to the meshing theory, the normal vector is perpendicular to the relative motion vector at the meshing point. Namely, the product of the normal vector and the relative motion vector is zero as shown in Eq. (13).

$$
\Phi=\boldsymbol{n} \cdot \boldsymbol{v}=0
$$

a. The relative velocity at the meshing point.

According to Fig. 2, the unit vectors satisfy the following condition among $S_{d}, S_{f}$ and $S_{1}$.

$$
-\boldsymbol{j}_{d}=\boldsymbol{k}_{f}=\boldsymbol{k}_{1}
$$

And Eq. (9) could be rewritten as

$$
\boldsymbol{r}_{d}^{(d)}=-\left( \pm u \sin \alpha+R_{T} \pm \frac{m \pi}{4}\right) \cos \theta \boldsymbol{i}_{d}+\left( \pm u \sin \alpha+R_{T} \pm \frac{m \pi}{4}\right) \sin \theta \boldsymbol{j}_{d}+u \cos \alpha \boldsymbol{k}_{d}
$$

The relative velocity can be represented as

$$
\boldsymbol{v}_{d}^{(d 1)}=\boldsymbol{v}_{d}^{(d)}-\boldsymbol{v}_{d}^{(1)}=\left(\boldsymbol{\omega}_{d}^{(d)} \times \boldsymbol{r}_{d}^{(d)}+\boldsymbol{v}_{d}\right)-\left(\boldsymbol{\omega}_{d}^{(1)} \times \boldsymbol{r}_{d}^{(d)}+\boldsymbol{E}_{d} \times \boldsymbol{\omega}_{d}^{(1)}\right)
$$

where $\boldsymbol{E}_{d}=-\left(R_{1} \pm R_{1} \varphi_{1}\right) \boldsymbol{i}_{d}-R_{1} \boldsymbol{k}_{d}, \boldsymbol{\omega}_{d}^{(d)}=0, \boldsymbol{\omega}_{d}^{(1)}=-\omega_{1} \boldsymbol{k}_{1}=\omega_{1} \boldsymbol{j}_{d}, \boldsymbol{v}_{d}=\omega_{1} R_{1} \boldsymbol{i}$.

Then, Eq. (16) could be rewritten as

$$
\boldsymbol{v}_{d}^{(d 1)}=-\omega_{1} u \cos \alpha \boldsymbol{i}_{d}-\omega_{1}\left[\left( \pm u \sin \alpha+R_{T} \pm \frac{m \pi}{4}\right) \cos \theta-\left(R_{T}+R_{1} \varphi_{1}\right)\right] \boldsymbol{k}_{d}
$$

b. The contact trace equation between the cutter and the workpiece.

Substituting Eq. (12) and Eq. (17) into Eq. (13), the condition which $u$ should satisfy can be represented as

$$
u=\mp \frac{\sin \alpha}{\cos \theta}\left[\cos \theta\left(R_{T} \pm \frac{m \pi}{4}\right)-\left(R_{T}+R_{1} \varphi_{1}\right)\right]
$$

Therefore, combining Eq. (8) and Eq. (18), the contact trace equation is represented as

$$
\left\{\begin{array}{l}
x_{d}=-\left( \pm u \sin \alpha+R_{T} \pm \frac{m \pi}{4}\right) \cos \theta \\
y_{d}=\left( \pm u \sin \alpha+R_{T} \pm \frac{m \pi}{4}\right) \sin \theta \\
z_{d}=u \cos \alpha \\
u=\mp \frac{\sin \alpha}{\cos \theta}\left[\cos \theta\left(R_{T} \pm \frac{m \pi}{4}\right)-\left(R_{T}+R_{1} \varphi_{1}\right)\right]
\end{array}\right.
$$


c. The tooth surface equation.

The tooth surface equation can be represented through the coordinate transformation.

$$
\left[\begin{array}{llll}
x_{1} & y_{1} & z_{1} & 1
\end{array}\right]^{T}=M\left[\begin{array}{llll}
x_{d} & y_{d} & z_{d} & 1
\end{array}\right]^{T}
$$

It could be rewritten as

$$
\left\{\begin{array}{l}
x_{1}=\left[-\left( \pm u \sin \alpha+R_{T} \pm \frac{\pi m}{4}\right) \cos \theta+R_{T}+R_{1} \varphi_{1}\right] \cos \varphi_{1}-\left(R_{1}+u \cos \alpha\right) \sin \varphi_{1} \\
y_{1}=\left[-\left( \pm u \sin \alpha+R_{T} \pm \frac{\pi m}{4}\right) \cos \theta+R_{T}+R_{1} \varphi_{1}\right] \sin \varphi_{1}+\left(R_{1}+u \cos \alpha\right) \cos \varphi_{1} \\
z_{1}=-\left( \pm u \sin \alpha+R_{T} \pm \frac{\pi m}{4}\right) \sin \theta \\
u=\mp \frac{\sin \alpha}{\cos \theta}\left[\cos \theta\left(R_{T} \pm \frac{\pi m}{4}\right)-\left(R_{T}+R_{1} \varphi_{1}\right)\right]
\end{array}\right.
$$

\subsection{Tooth surface reconstruction and 3D model}

At present, the method of modelling CATT gears is not accurate or clear for the rotating knife dish milling. In the literature $[9,23]$ a method is presented to create a $3 \mathrm{D}$ model of CATT gear by the simulation processing using the gear rack, but then there are knife marks on the tooth surface and the 3D model cannot be used for further study like the finite element analysis and machining. In [10] a new method for creating a 3D model is given, but the modelling process is not clear, and the reference value is not high. Therefore, a new modelling method is discussed to realize the tooth surface reconstruction and create an accurate $3 \mathrm{D}$ model.

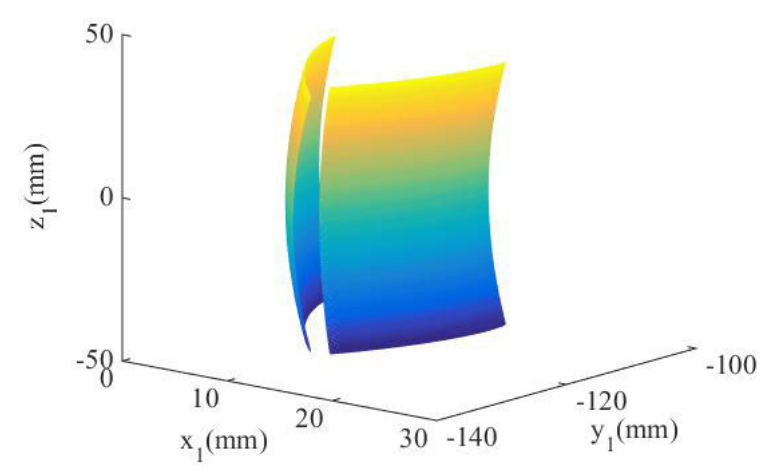

Fig. 4 Single-tooth surface model

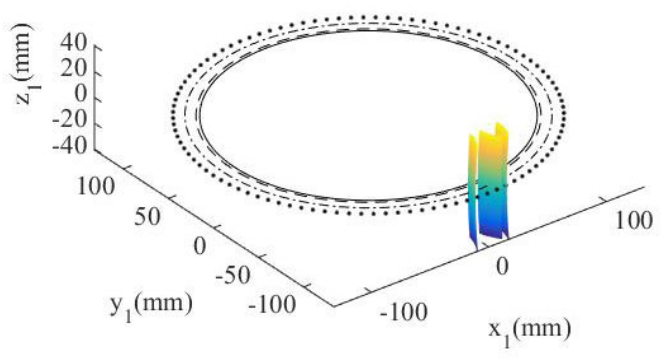

Fig. 5 Multi-tooth surface model

Based on the tooth surface equation, pictures of the tooth surface model are obtained by the MATLAB simulation shown in Fig. 4 and Fig. 5. The parameters of the CATT gear are as follows: $m=8 \mathrm{~mm} ; z=31 ; R_{T}=500 \mathrm{~mm} ; b=80 \mathrm{~mm}$. Fig. 4 presents a single-tooth surface model. Fig. 5 presents a multi-tooth surface model. To create the accurate 3D model further, the point cloud of the tooth surface is output and imported into UG to create gear surface shown in Fig. 6. Applying corresponding commands, an accurate 3D model is created and assembled as shown in Fig. 7. The parameters of the CATT gear are as follows: $m=8 \mathrm{~mm} z=19$; $R_{T}=500 \mathrm{~mm} ; b=80 \mathrm{~mm}$. By comparing the CATT gear's surface quality of this model and the model presented in the literature $[9,23]$, there are many knife marks on the surface of the model presented in the literature, however the model created in this paper has a high surface quality as shown in Fig. 8. It can be further used to study the finite element analysis and machining. 


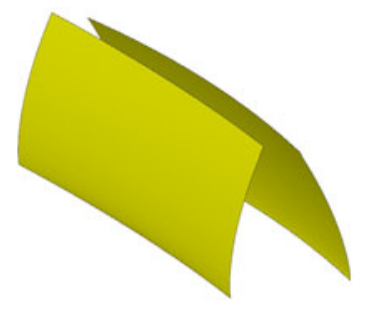

Fig. 6 Tooth surface model

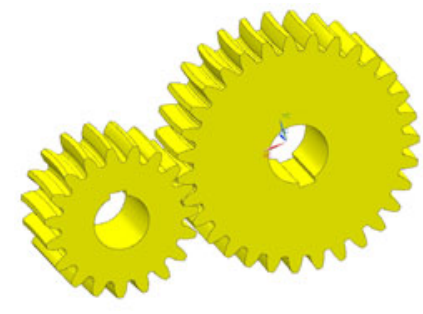

Fig. 7 Assembled model of the CATT gear

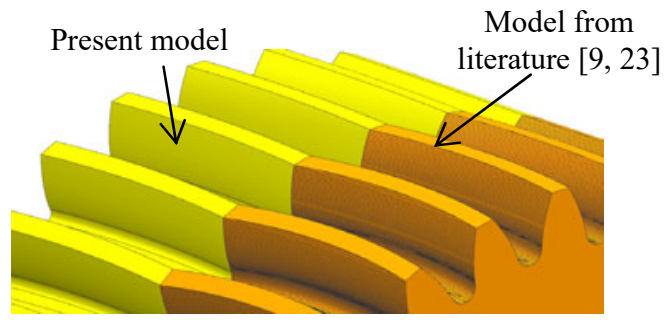

Fig. 8 Comparison of the two gear models

\subsection{Tooth profile equation}

The paper [7] indicates that an ideal CATT gear has equal tooth space width and tooth thickness, and the gear's whole tooth profile is involute. But as for the CATT gear cut by the rotating knife dish, the tooth profile differs from ideal CATT gears. The paper [11] studied the tooth surface curvature characteristics of a cylindrical gear and pointed out features of the tooth profile, but the study is not deep, there is no rigorous proof to support the conclusion. However, the characteristics of the tooth profile are very important to study the design, profile modification, lubrication and measuring of gears. Therefore, it is necessary to prove definitively the tooth profile of CATT gears by applying the mathematical method.

To study the tooth profile of the CATT gear in each cross section, the tooth width is assumed as $b$. Therefore, the $z$ coordinate of the tooth surface in the $S_{1}$ can be represented as:

$$
z_{1}=-\left( \pm u \sin \alpha+R_{T} \pm \frac{m \pi}{4}\right) \sin \theta=b
$$

If $b \neq 0, \theta \neq 0, \varphi_{1}$ can be represented as

$$
\varphi_{1}=-\frac{1}{R_{1}}\left\{\left[\frac{b}{\sin \theta}+\cos ^{2} \alpha\left(R_{T} \pm \frac{m \pi}{4}\right)\right] \frac{\cos \theta}{\sin ^{2} \alpha}+R_{T}\right\}
$$

so the tooth profile equation of in the arbitrary cross section can be represented as

$$
\left\{\begin{array}{l}
x_{1}=\left[-\left( \pm u \sin \alpha+R_{T} \pm \frac{\pi m}{4}\right) \cos \theta+R_{T}+R_{1} \varphi_{1}\right] \cos \varphi_{1}-\left(R_{1}+u \cos \alpha\right) \sin \varphi_{1} \\
y_{1}=\left[-\left( \pm u \sin \alpha+R_{T} \pm \frac{\pi m}{4}\right) \cos \theta+R_{T}+R_{1} \varphi_{1}\right] \sin \varphi_{1}+\left(R_{1}+u \cos \alpha\right) \cos \varphi_{1} \\
\varphi_{1}=-\frac{1}{R_{1}}\left\{\left[\frac{b}{\sin \theta}+\cos ^{2} \alpha\left(R_{T} \pm \frac{m \pi}{4}\right)\right] \frac{\cos \theta}{\sin ^{2} \alpha}+R_{T}\right\} \\
u=\mp \frac{\sin \alpha}{\cos \theta}\left[\cos \theta\left(R_{T} \pm \frac{\pi m}{4}\right)-\left(R_{T}+R_{1} \varphi_{1}\right)\right]
\end{array}\right.
$$


In the middle cross section, $b=0, \theta=0$, the tooth profile equation can be represented as

$$
\left\{\begin{array}{l}
x_{1}=\left(R_{1} \varphi_{1} \cos \alpha \mp \frac{\pi m}{4} \cos \alpha \pm R_{1} \sin \alpha\right) \cos \left(\varphi_{1} \pm \alpha\right)-R_{1} \sin \left(\varphi_{1} \pm \alpha\right) \cos \alpha \\
y_{1}=\left(R_{1} \varphi_{1} \cos \alpha \mp \frac{\pi m}{4} \cos \alpha \pm R_{1} \sin \alpha\right) \sin \left(\varphi_{1} \pm \alpha\right)+R_{1} \cos \left(\varphi_{1} \pm \alpha\right) \cos \alpha
\end{array}\right.
$$

As for the involute profile, $R_{b 1}=R_{1} \cos \alpha$ and $R_{1} \sin \alpha=R_{b 1} \tan \alpha=R_{b 1} \theta_{K}+R_{b 1} \alpha, R_{b 1}$ is the radius of the base circle, $\theta_{K}$ is the involute opening angle.

Eq. (25) can also be rewritten as

$$
\left\{\begin{array}{l}
x_{1}=R_{b 1}\left(\varphi_{1} \pm \alpha\right) \cos \left(\varphi_{1} \pm \alpha\right)-R_{b 1} \sin \left(\varphi_{1} \pm \alpha\right) \\
y_{1}=R_{b 1}\left(\varphi_{1} \pm \alpha\right) \sin \left(\varphi_{1} \pm \alpha\right)+R_{b 1} \cos \left(\varphi_{1} \pm \alpha\right)
\end{array}\right.
$$

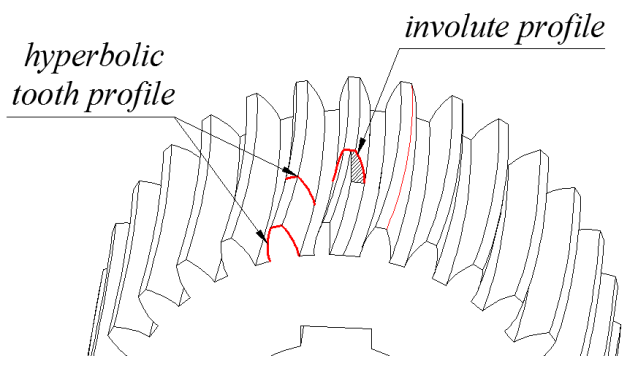

Fig. 9 Tooth profile of CATT gear

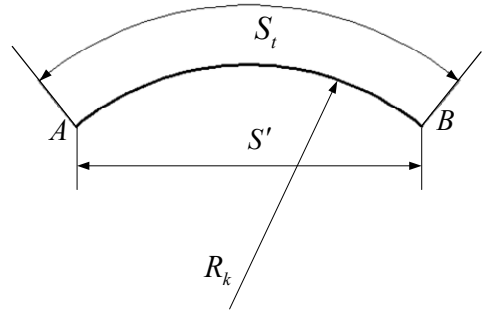

Fig. 10 Tooth thickness of arbitrary cross section

Eq. (26) is the involute profile parametric equation. It indicates that the tooth profile of the CATT gear is an involute tooth profile in the middle cross section. In the other arbitrary cross section, when the parameters of the cutter and the workpiece are determined, the tooth profile equation is a single parameter equation about $\theta$, so the tooth profile is not an involute profile. According to [24], Eq. (24) is a hyperbolic equation. Namely, the tooth profile of the other arbitrary cross section is a hyperbolic tooth profile.

\subsection{Tooth thickness calculation}

Meanwhile, the tooth thickness of a CATT gear has also a great influence on the design, profile modification, lubrication, strength and measuring of gears. Therefore, the tooth profile and the tooth thickness must be studied in depth. The tooth thickness of the CATT gear is divided into two categories: normal tooth thickness $S_{n}$ and circumferential tooth thickness $S_{t}$. This paper mainly discusses the circumferential tooth thickness.

The tooth thickness of the arbitrary cross section is shown schematically in Fig. 10. The arc length between point $A$ and point $B$ is the tooth thickness. If the coordinates of $A$ and $B$ are known, $S_{t}$ can be calculated by Eq. (27).

$$
\left\{\begin{array}{l}
S^{\prime}=\sqrt{\left(x_{A}-x_{B}\right)^{2}+\left(y_{A}-y_{B}\right)^{2}} \\
S_{t}=2 R_{k} \arcsin \left(\frac{S^{\prime}}{2 R_{k}}\right)
\end{array}\right.
$$

where $\left(x_{A}, y_{A}\right)$ are the coordinates of point $A,\left(x_{B}, y_{B}\right)$ are the coordinates of point $B, S^{\prime}$ is the chord length, $R_{k}$ is the arbitrary circular radius between the tooth top and the base circle.

Based on the tooth profile equation, pictures of the tooth profile are obtained from MATLAB simulation shown in Fig. 11 and Fig. 12. The parameters of the CATT gear are as follows: $m=8 \mathrm{~mm} ; z=31 ; b=80 \mathrm{~mm} ; R_{T}=200 \mathrm{~mm}$ and $300 \mathrm{~mm}$, respectively. 


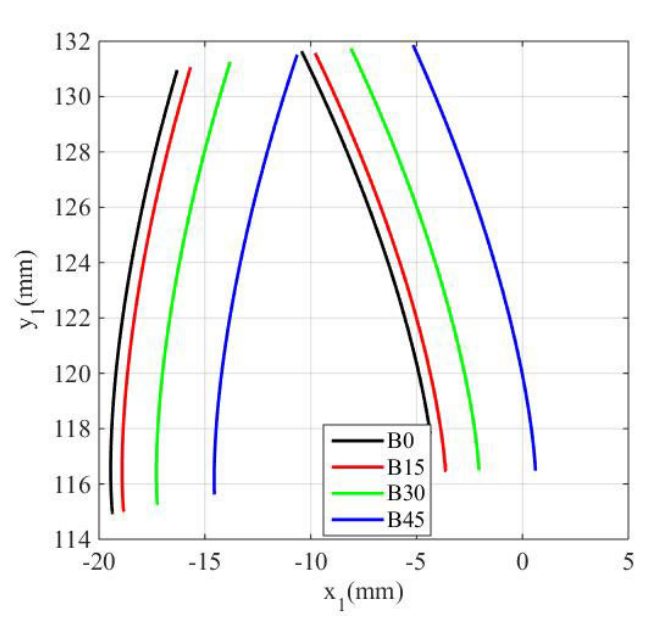

Fig. 11 Tooth profile $R_{T}=200 \mathrm{~mm}$

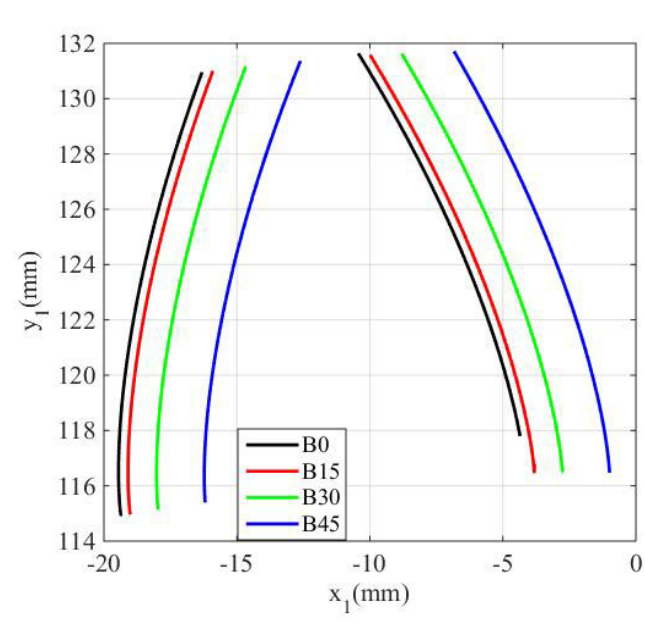

Fig. 12 Tooth profile $R_{T}=300 \mathrm{~mm}$

\section{Discussion}

According to the tooth profile equation, the tooth profile of the middle cross section is an involute profile, and the tooth profile of the other arbitrary cross section is a hyperbolic tooth profile. The designing parameters including $R_{T}, b$, and $m$ etc. have a great influence on the tooth profile shape. Therefore, the tooth thickness is different in different cross sections.

In addition, the study [11] indicates that the contact form of the CATT gear in case of the rotating knife dish milling process is a point contact, and the area of the contact ellipse increases as external loads increase. Therefore, it is feasible to improve the bearing ability of the gear by modifying the tooth surface profile. But the tooth profile equation would change as the gear width $b$ changes, so the modification quantity is uncertain. However, the paper [16] does not offer a method to solve the effect of gear width on the modification quantity. The discussion can provide a foundation for the tooth surface modification.

Fig. 13 and Fig. 14 show the influence of the gear width on the tooth thickness. Fig. 15 and Fig. 16 show the influence of the tooth line radius on the tooth thickness. Fig. 17 and Fig. 18 show the influence of the module on the tooth thickness. In the figures, B0 expresses the middle cross section; B15, B30 and B45 mean that the distance from the measured cross section to the middle cross section is $15 \mathrm{~mm}, 30 \mathrm{~mm}$, and $45 \mathrm{~mm}$, respectively; $e$ expresses the tooth thickness deviation, which is equal to the tooth thickness of the measured cross section minus the tooth thickness of the middle cross section; the range of the radius $R_{k}$ extends from the basic radius $R_{b 1}$ to the gear top circle radius $R_{a}$.

\subsection{Influence of the gear width on the tooth thickness}

The gear width is an important parameter of gear design, and it has an important influence on the geometry characteristics of the tooth surface and contact performances of CATT gears. Fig. 13 and Fig. 14 show the influence of the gear width on the tooth thickness. The parameters are as follows: $m=4 \mathrm{~mm} ; z=31 ; R_{T}=100 \mathrm{~mm}, 200 \mathrm{~mm}, 300 \mathrm{~mm}$ and $400 \mathrm{~mm}$; the gear width is B0, B15, B30 and B45. 
a)

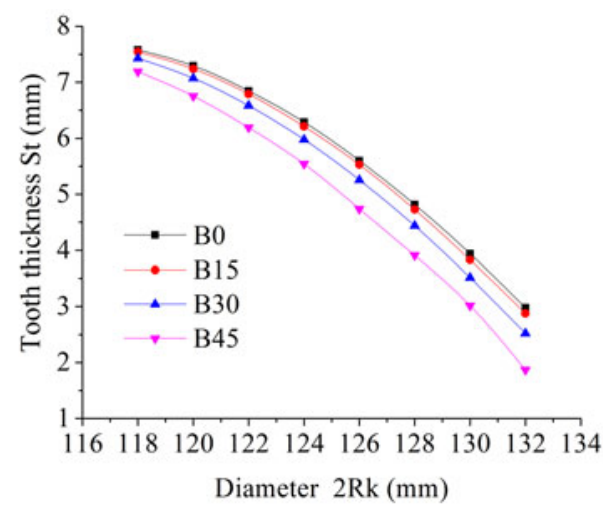

c)

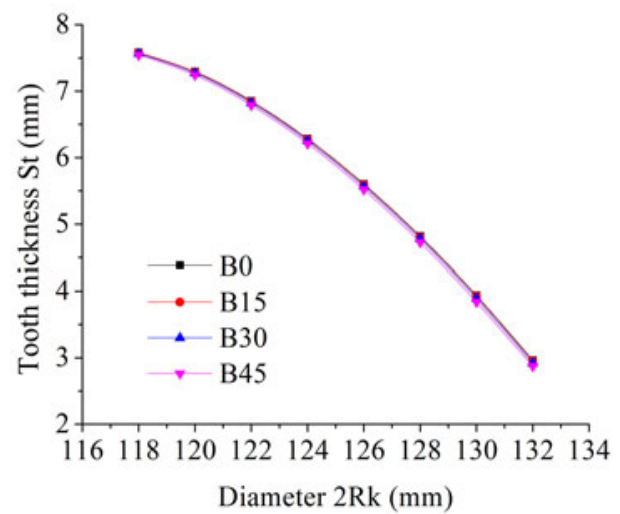

b)

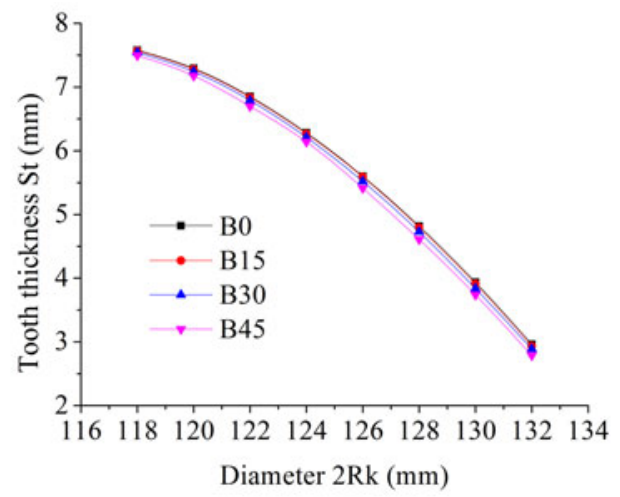

d)

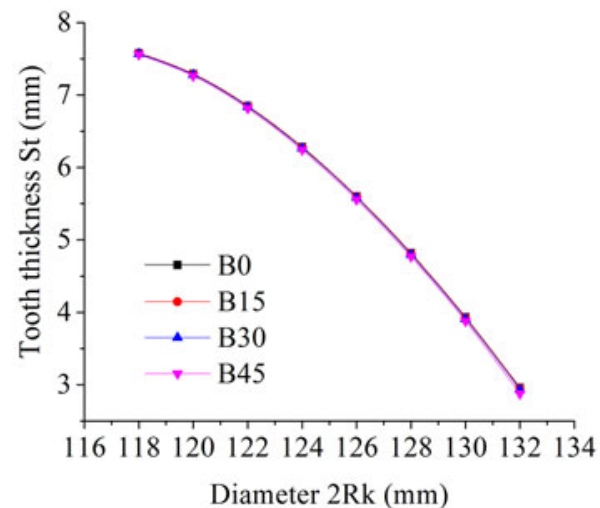

Fig. 13 Influence of $b$ on $S t$, a) $R_{T}=100$, b) $R_{T}=200$, c) $R_{T}=300$, d) $R_{T}=400$

a)

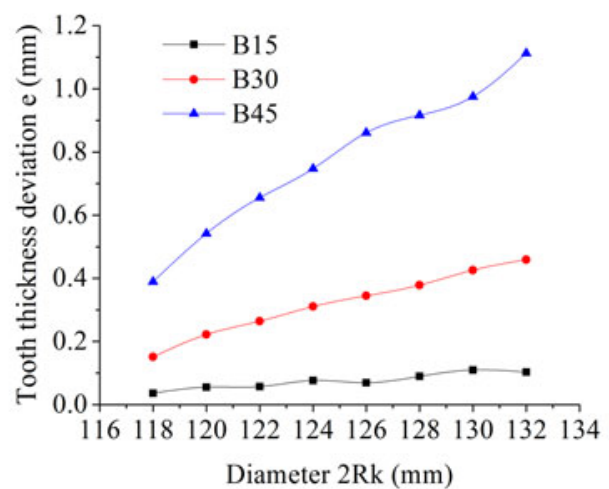

c)

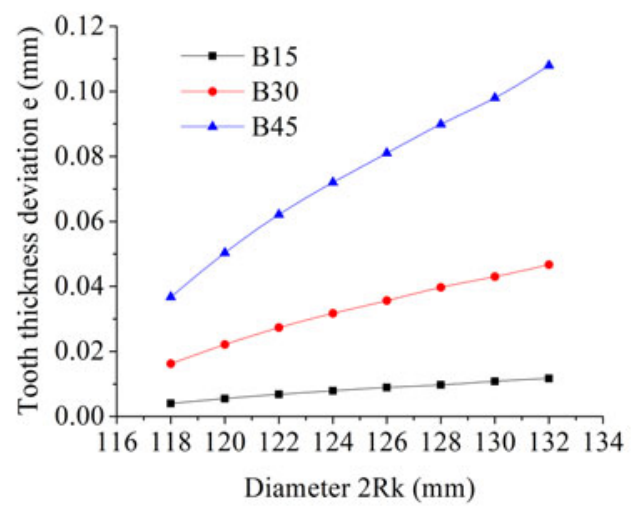

b)

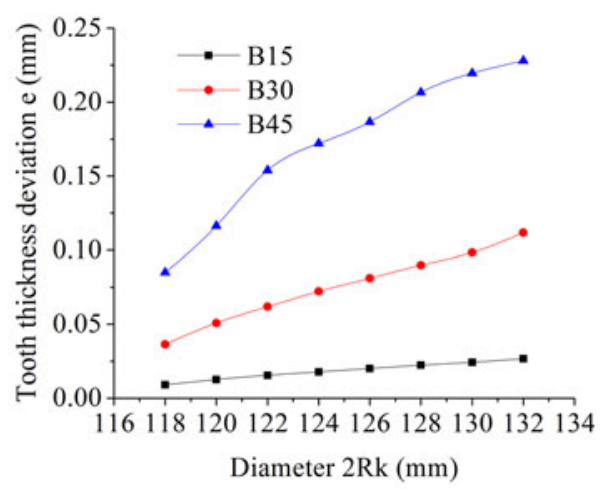

d)

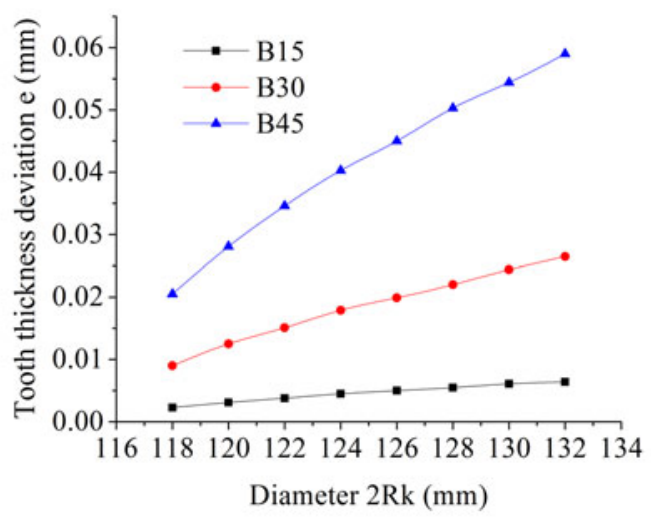

Fig. 14 Influence of $b$ on $e$, a) $R_{T}=100$, b) $R_{T}=200$, c) $R_{T}=300$, d) $R_{T}=400$

It is obvious from the graphs that the gear width has an effect on the tooth thickness, and the tooth thickness deviation increases with an increase in the gear width. This indicates 
that the tooth thickness gradually becomes thin from the middle cross section to the end surface. It results in low bearing capacity. Meanwhile, due to the thin gear tooth, the tooth surface at the end face will not establish contact; it can result in some waste of material if the gear width is greater. Moreover, at the same cross section, the tooth thickness deviation increases as $R_{k}$ increases. That is to say, the higher the tooth height is, the thinner the tooth thickness becomes. But the closer the cross section is, the smaller the tooth thickness deviation becomes.

\subsection{Influence of the tooth line radius on the tooth thickness}

The tooth line radius is also an important parameter of CATT gears, and it has an important influence on the geometry characteristics of the tooth surface and contact performances of CATT gears. The parameters of the CATT gear are the same as in Section 3.1.

Fig. 15 shows the influence of the tooth line radius on the tooth thickness. From the graphs, it is obvious that the tooth line radius affects the tooth thickness. At the middle cross section, the tooth line radius has almost no influence on the tooth thickness. The tooth profile equation of the middle cross section Eq. (26) indicates that Eq. (26) is not a parametric equation of the tooth line radius. The simulation results are consistent with Eq. (26). In the other arbitrary cross section, as the tooth line radius increases, the tooth thickness becomes thinner. When the tooth line radius is close to the gear width, the tooth profiles of the concave and the convex tooth surface tend to intersect as shown in Fig. 16. This indicates that CATT gear cannot be cut completely when the tooth line radius is close to or less than the gear width. Moreover, Fig. 14 shows that the tooth thickness deviation will increase as the tooth line radius increases with the same parameters.

a)

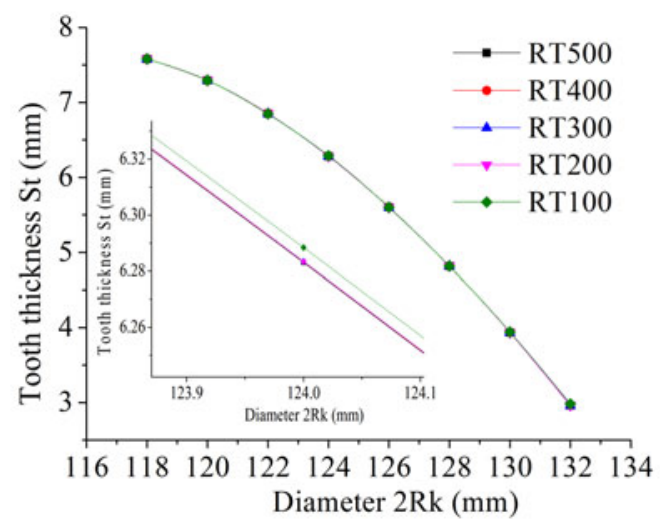

c)

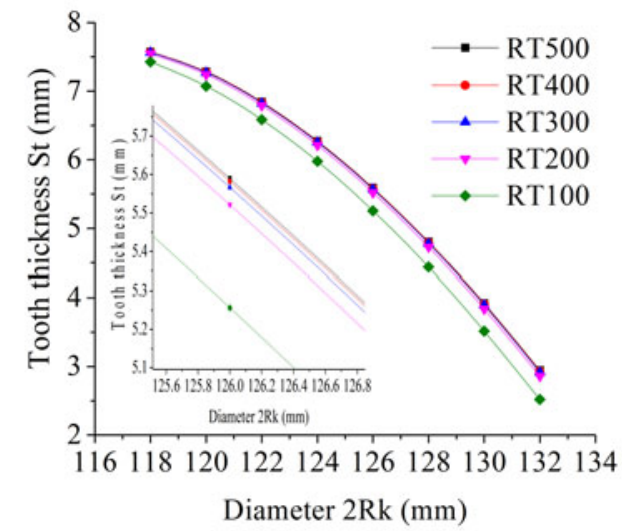

b)

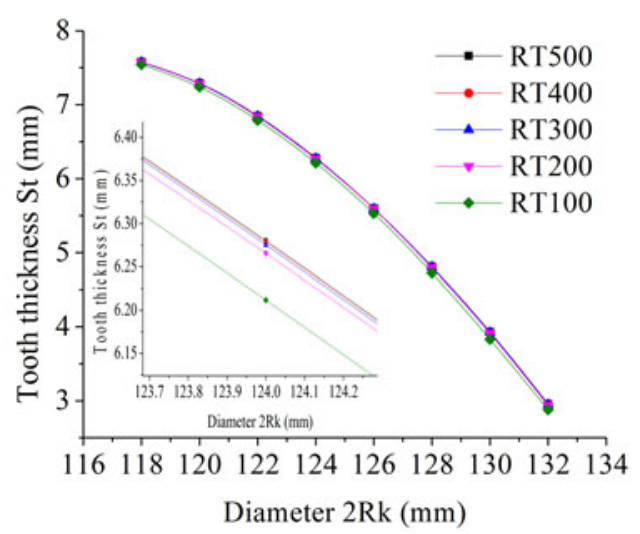

d)

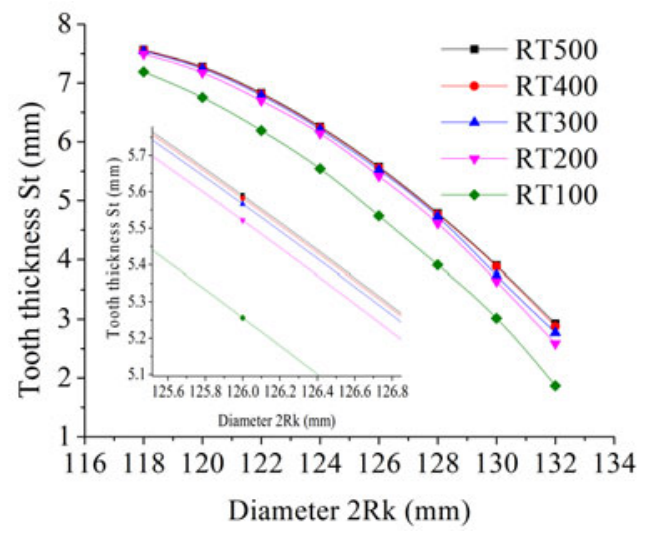

Fig. 15 Influence of $R_{T}$ on $S_{t}$, a) B0,b) B15,c) B30,d) B45 
a)

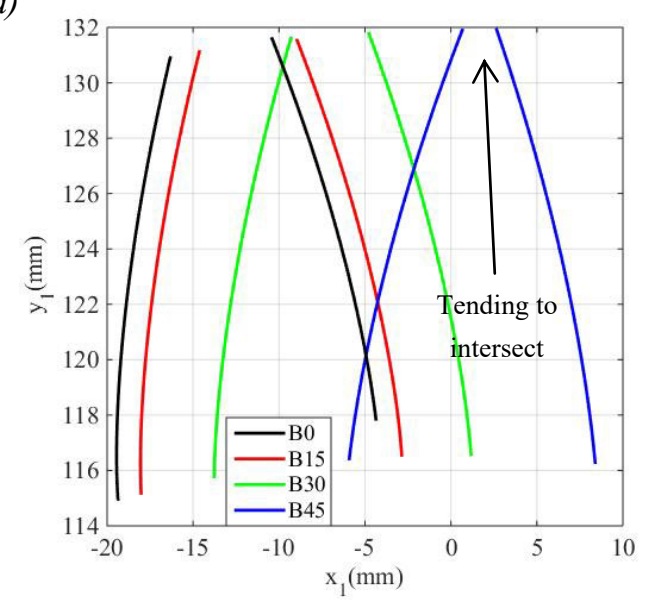

b)

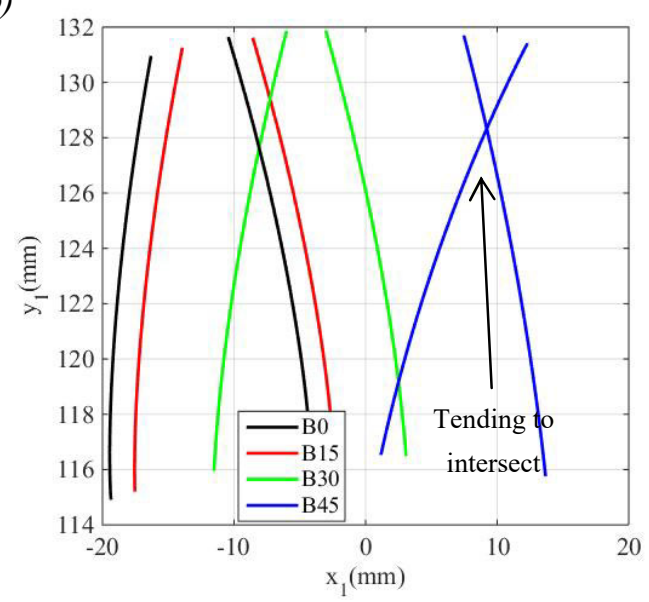

Fig. 16 Tooth surfaces tend to intersect, a) $R_{T}=80$, b) $R_{T}=60$

\subsection{Influence of the module on the tooth thickness}

Module is an important parameter of the gear design. It also has a great influence on the tooth profile. Fig. 17 and Fig. 18 show the influence of the module on the tooth thickness. The module of the CATT gear is 6 and 8. From Fig. 14, Fig. 17 and Fig. 18, it is obvious that the tooth thickness deviation increases as the module increases. But the relative tooth thickness deviation, namely $e$ divided by the tooth profile of the middle cross section, decreases as the module increases.

a)

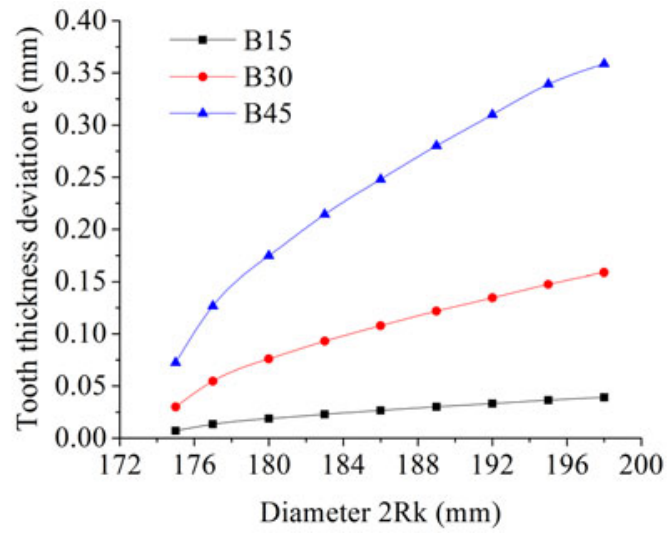

b)

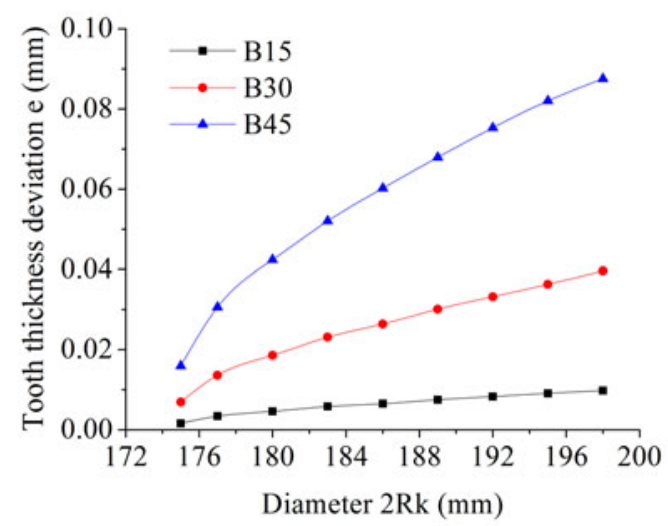

Fig. 17 Influence of $m$ on $e, \mathrm{~m}=6 \mathrm{~mm}$, a) $R_{T}=200$; b) $R_{T}=400$

Increasing the gear width results in the improvement of the bearing capacity in practical application, and this is feasible for the spur gear and helical gear. As for the CATT gear, based on the influence of the tooth line radius, the gear width and the module on the tooth profile, the tooth width cannot be too large and is constrained by the tooth line radius, because the tooth thickness deviation increases with an increase in the gear width under the fixed tooth line radius. According to Sections 3.1 and 3.2, the authors suggest that the tooth line radius should be three or four times wider than the tooth width to ensure the contact section as large as possible in the direction of the gear width. Moreover, the module should be large to improve stiffness and stability. 

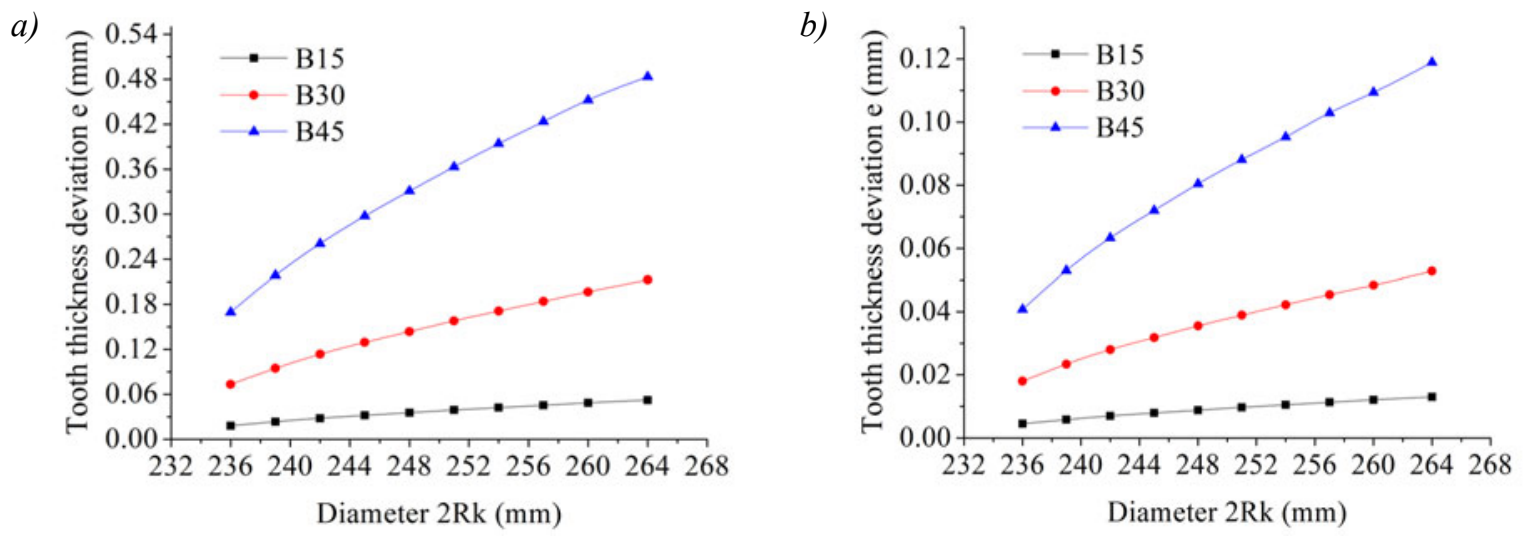

Fig. 18 Influence of $m$ on $e, \mathrm{~m}=8 \mathrm{~mm}$, a) $R_{T}=200$; b) $R_{T}=400$

\section{Conclusions}

This paper discusses the tooth surface reconstruction and the tooth profile geometry of CATT gears. Firstly, based on the space meshing theory and the principles of differential geometry, the tooth surface equation of CATT gears was deduced. Next, the tooth surface of a CATT gear was reconstructed and a 3D model was created. Then, the tooth profile equation and tooth thickness calculation formula for CATT gears were deduced according to the tooth surface equation. Finally, the influences of the design parameters on the tooth profile were investigated. The main conclusions can be expressed as follows:

1. By theoretical deduction it is proven that the profile of the middle section is involute, and the other section is hyperbolic.

2. The results of the analysis of influences of the design parameters on the tooth profile show that the gear width, the tooth line radius and the module have a great influence on the tooth profile. The general tendency is that the gear tooth thickness becomes thinner with an increase in the gear width and a decrease in the tooth line radius, and the tooth profiles of the concave and the convex tooth surface may intersect. The influences of the module need to be discussed separately. The tooth thickness deviation increases as the module increases. However, the relative tooth thickness deviation decreases as the module increases.

3. Based on the above conclusions, the authors suggest that the tooth line radius should be three or four times wider than the tooth width and the module should be large to improve bearing capacity.

\section{Acknowledgement}

This project is supported by the Guizhou Province Education Department Young Science and Technology Talents Growth Project (Qianjiaohe KY Zi [2018] 319), the Zunyi Science and Technology Bureau and the Zunyi Normal University Joint Science and Technology Fund Project (Zunshi Kehe [2018] 13) and the Guizhou High Level Innovative Talent Training Program ([2016] 9).

\section{REFERENCES}

[1] Miler D, Lončar A, Žeželj D, Domitran, Z. Influence of profile shift on the spur gear pair optimization. Mechanism and Machine Theory 117 (2017) 189-197.

https://doi.org/10.1016/j.mechmachtheory.2017.07.001 
[2] Ghosh S S, Chakraborty G. On optimal tooth profile modification for reduction of vibration and noise in spur gear pairs. Mechanism and Machine Theory 105 (2016) 145-163. https://doi.org/10.1016/j.mechmach-theory.2016.06.008

[3] Tseng J T, Tsay C B. Mathematical model and surface deviation of cylindrical gears with curvilinear shaped teeth cut by a hob cutter. Journal of Mechanical Design 127 (5) (2005) 271-277. https://doi.org/10.1115/1.1876437

[4] Peng Y, Song A, Shen Y, Lin, X. A novel arc-tooth-trace cycloid cylindrical gear. Mechanism and Machine Theory 118 (2017) 180-193. https://doi.org/10. 1016/j. mechmachtheory.2017.08.009

[5] Chen Zongmin, Hou Li., Duan Yang. Analysis of nonlinear vibration on a cylindrical gear with variational hyperbola and circular arc tooth trace. Advanced Engineering Sciences 49 (2) (2016) 209-216. https://doi.org/10.15961/j.jsuese. 201600806

[6] Yan Lili, Hou Li, You Yunxia. Study on forming principle and the maximum contact stress of circulararc-tooth-trace cylinder gear. Journal of Mechanical Trans-mission 38 (8) (2014) 26-29. https://doi.org/10.165 78/j.issn.1004.2539.2014.08.028

[7] Zhang Qi, Hou Li, Tang Rui. Method of processing and an analysis of meshing and contact of circular arc tooth trace cylindrical gears. Transactions of Famena 40 (4) (2017) 11-24. https://doi.org/10.21278/TOF.40402

[8] Chang Qinglin, Hou Li. Parallel translating mechanism process oriented mathematical model and 3-D Model for cylindrical gears with curvilinear shaped teeth. Jordan Journal of Mechanical and Industrial Engineering 10 (3) (2017) 171-177.

[9] Wang Shaojiang, Hou Li, Dong Lu. Modeling and strength analysis of cylindrical gears with curvilinear shape teeth for manufacture. Journal of Sichuan University 44 (2) (2012) 210-215. https://doi.org/10.15961/j.jsues e.2012.02. 029

[10] Xiao Huajun, Hou Li, Dong Lu. Mathematical modeling of rotary cutter arc tooth line of cylindrical gear shaped by origin face of rotary cutter. Journal of Sichuan University 45 (3) (2013) 197-199. https://doi.org/10.15 961/j.jsuese.2013.03.019

[11] Wei Yongqiao, Ma Dengqiu, Wu Yang Study on the tooth surface and curvature characteristics of cylindrical gear with variable hyperbolic arc tooth trace. Advanced Engineering Sciences 49 (6) (2017) 196-203. https://doi.org/10.15961/j.jsuese.201700372

[12] Rui-Tang T., Chung-Biau T.. Contact characteristics of cylindrical gears with curvilinear shaped teeth. Mechanism and Machine Theory 39 (4) (2004) 905-919. https://doi.org/10.1016/j.mechmachtheory.2004.04.006

[13] Litvin, F. L., Tsay, C. B. Helical gears with circular arc teeth: simulation of conditions of meshing and bearing contact. Journal of Mechanical Design 107 (4) (1985) 556-564. https://doi.org/10.1115/1.3260763

[14] Litvin F.L., Alfonso F., Ignacio G.P. Modified involute helical gears: Computerized design, simulation of meshing and stress analysis. Computer Methods in Applied Mechanics and Engineering 192 (33-34) (2003) 3619-3655. https://doi.org/10.1016/S00 45-7825(03)00367-0

[15] Di Y, Hong X, Chen M.. Generation principle of arcuate tooth trace cylindrical gear. Journal of Harbin Bearing 27 (3) (2006) 58-61.

[16] Ma Zhenqun, Deng Chengyi: CNC machining method of whole modified surface of cylindrical gears with arcuate tooth trace, Journal of Mechanical Engineering 48 (5) (2012) 165-171. https://doi.org/10.3901/JME.2012.05.165

[17] Jiang Weixu, Hou Li, Zhang Jianquan. The feature modelling of gear with curvilinear tooth based on UG. Modular Machine Tool \& Automatic Manufacturing (12) (2010) 47-49.

[18] Fuentes-Aznar,A., Ruiz-Orzaez, R., Gonzalez-Perez, I. Comparison of spur, helical and curvilinear gear drives by means of stress and tooth contact analyses. Meccanica 52 (7) (2017) 1721-1738. https://doi.org/10.100 7/s110 12 -016-0515-y

[19] Zhang X, Xie Y, Tan X. Design, meshing characteristics and stress analysis of cylindrical gears with curvilinear tooth profile. Transactions of Famena 40 (1) (2016) 27-44.

[20] Chen, Y. C., C. C. Lo. Contact stress and transmission errors under load of a modified curvilinear gear set based on finite element analysis. ARCHIVE Proceedings of the Institution of Mechanical Engineers Part C Journal of Mechanical Engineering Science 203-210 (229.2) (2015):191-204. https://doi.org/10.1177/0954406214532907 
[21] Fuentes, A., Ruiz-Orzaez, R., Gonzalez-Perez, I. Computerized design, simulation of meshing, and finite element analysis of two types of geometry of curvilinear cylindrical gears. Computer Methods in Applied Mechanics \& Engineering 272 (2) (2014) 321-339. https://doi.org/0.1016/j.cma.2013.12.017

[22] Ma Dengqiu, Hou Li, Wang Hong. Modeling and dynamics analysis of circular arc tooth trace cylindrical gear or manufacturing. Machinery Design and Manufacture (11) (2017) 9-13.

[23] You Yunxia, Hou Li, Yan Lili. Modeling and contact analysis of circular arc tooth trace cylindrical gear for manufacture. Journal of Mechanical Transmission 39(6) (2015) 62-25. https://doi.org/10.16578/j.issn.1004.253 9 .2015.06.019

[24] Wu Daren, Luo Jiashun: The principle of gear meshing, Beijing: Science Press (1985) 113-118.

Submitted: $\quad 12.4 .2018$

Accepted: $\quad 19.10 .2018$
Dengqiu Ma (corresponding author)

Zhenhuan Ye

Yang Hang

School of Engineering and Technology, Zunyi Normal College, Zunyi, 56300, China

scumdq@163.com 